\title{
Emittance growth due to multipole transverse magnetic modes in an rf gun
}

\author{
M. S. Chae, ${ }^{1}$ J. H. Hong, ${ }^{1}$ Y. W. Parc, ${ }^{1, *}$ In Soo Ko, ${ }^{1}$ S. J. Park, ${ }^{2}$ H. J. Qian, ${ }^{3}$ W. H. Huang, ${ }^{3}$ and C. X. Tang ${ }^{3}$ \\ ${ }^{1}$ Department of Physics, Pohang University of Science and Technology, Pohang 790-784, Korea \\ ${ }^{2}$ Pohang Accelerator Laboratory, Pohang University of Science and Technology, Pohang 790-784, Korea \\ ${ }^{3}$ Department of Engineering Physics, Tsinghua University, Beijing 100084, China
}

(Received 25 March 2011; published 28 October 2011)

\begin{abstract}
Cylindrical asymmetry of an rf gun cavity excites the multipole rf field which will result in emittance growth of electron beams. With demanding of electron beams of ultralow emittance for the x-ray free electron laser, it is necessary to study the effect of the multipole rf field to the emittance growth and to eliminate the multipole field effect on electron beam emittance in an rf gun. In this paper, we present both theoretical analysis and numerical simulations of the multipole field effect to the emittance growth. We proposed a four-hole scheme to minimize the emittance growth from the multipole field.
\end{abstract}

PACS numbers: 41.75.- i, 41.85.- $\mathrm{p}$

\section{INTRODUCTION}

In a photocathode radio frequency (rf) gun, time dependent transverse deflection of each electron due to the Lorentz force in the cavity can induce transverse emittance growth [1]. Besides the intrinsic kick from the fundamental monopole mode $\left(\mathrm{TM}_{010}\right)$, multipole modes due to asymmetric boundary conditions of the cavity wall further degrade the beam emittance. Certain ports, such as laser ports, vacuum ports, and power-coupling ports, break the cylindrical symmetry in different cells of the photocathode rf gun, and the primary asymmetry usually lies in the cell with the biggest port [2]. Nonaxisymmetric structure excites multipole field components, such as the dipole, quadrupole, and octopole, which kick the beam time dependently, leading to the emittance growth [1]. While removal of the kick from the fundamental mode is difficult [3], minimization of the multipole rf field has been investigated and implemented in the design of the state-ofthe-art photocathode if gun $[4,5]$, which aims to produce high-brightness electron beams.

The third-generation BNL-type rf gun was designed to feed rf power into the rf gun by a single coupling hole $[5,6]$. However, the one-sided coupling scheme causes asymmetry in the electromagnetic field inside the cavity. This asymmetric field can be expanded into multipole fields $[4,7]$. In particular, the dipole and quadrupole fields are the main causes of emittance growth. To reduce these multipoles, many innovative ideas were introduced. The Linac Coherent Light Source (LCLS) gun is one of the rf guns which many of these ideas were applied to $[5,8,9]$ and it showed a successful result $[5,10]$.

\footnotetext{
*young1@ postech.ac.kr
}

Published by the American Physical Society under the terms of the Creative Commons Attribution 3.0 License. Further distribution of this work must maintain attribution to the author(s) and the published article's title, journal citation, and DOI.
Pohang Accelerator Laboratory (PAL) has developed an S-band rf gun over the past six years [11-14]. In PAL, the four-holes model to reduce quadrupole mode is studied [12], the two additional holes in Ref. [12] play the same role as the racetrack shape in the LCLS gun [7]. Besides, the usage of two additional holes as pumping ports will increase pumping speed; thus, it is very advantageous to maintain a low vacuum level. In the four-hole scheme, by changing the dimension of the additional holes, the amplitude of dipole field can be successfully eliminated [12].

The authors in Ref. [12] have proposed a method to reduce the quadrupole field by making four holes in the full cell. They studied the whole effect of the multipole mode on the emittance growth. However, the effect of each mulipole field on the emittance growth is not studied in the paper. To understand the role of each multipole field in beam emittance degradation, an artificial rf field map which combines both fundamental monopole mode and one of the multipole fields must be used for beam dynamics simulations. In the simulation of the rf gun, there are too many factors concerning the emittance evolution, such as the space-charge effect, solenoid field, and so on. To understand the pure rf effect on the emittance growth, we need to simulate the electron beam propagation in a gun without space-charge effect or other elements.

In this paper, we have studied four gun models with zero, one, two, and four holes at the full cell cavity, respectively. Electromagnetic field distribution in the rf gun cavity can be obtained by using many simulation tools such as 3D high frequency eigenmode solvers [15]. After obtaining the rf field distributions of the four models, we manipulated the field map using the Fourier transform technique. We were able to control the amplitude of each multipole field (TM mode) to construct a field map as an input field for the phase and radial motion in the electron linacs (PARMELA) code [16]. The effect of the number of holes on the emittance growth was investigated by comparing the emittance evolution that used the original field map from each gun 
model with the simulation result that used the artificial field map with a single multipole component.

This paper is organized as follows. The formulas to calculate the emittance growth from each multipole field in the full cell are derived in Sec. II. The simulation models and the rf field manipulation are explained in Sec. III. The simulation results are presented in Sec. IV, and the conclusion and discussion are provided in Sec. V.

\section{THEORY}

In this section, we simplify our consideration to an electron beam passing through a single cell (i.e., the full cell in a BNL/LCLS-type gun) near the axis at the speed of light and evaluate the multipole field effect on beam qualities analytically. Along the number of the holes in the full cell of the rf gun, the dominant rf multipole fields are changed from dipole to quadrupole and finally octopole, in turn. Thus, only the effects of these multipoles on emittance growth are analyzed in this study.

The transverse momentum imparted to an electron beam by the rf field in a single cell is calculated by the PanofskyWenzel theorem $[17,18]$ as follows:

$$
p_{\perp}=\frac{e}{\omega} \operatorname{Re}\left(\int_{-L / 2}^{L / 2} i \nabla_{\perp} E_{z} d z\right),
$$

where $L$ is the cell length (i.e., half the rf wavelength), $E_{z}$ is the acceleration electric field along the longitudinal axis, which can be expressed as the sum of all the $\mathrm{TM}_{n 10}$ modes [7],

$E_{z}(r, \theta, z, t)=e^{i\left(\omega t+\varphi_{0}\right)} \sum_{n=0}^{\infty} E_{n} \cos (k z) J_{n}\left(k_{c} r\right) \cos \left[n\left(\theta-\theta_{0}\right)\right]$,

where $z$ and $r$ are longitudinal and radial coordinates, $\theta$ is azimuthal angle, $\theta_{0}$ is the polarization angle which will be determined by the position of holes, $\varphi_{0}$ is the rf phase, $k$ is the $\mathrm{rf}$ wave number $(2 \pi / \lambda), k_{c}$ is the radial wave number, and $J_{n}(x)$ is the Bessel function. In the case of the rf gun, the biggest hole is usually the rf coupling hole, and in the following derivations, the rf coupling hole is set to be in the $Y$ direction, so the polarization angle equals $\frac{\pi}{2}$.

The main mode is the monopole $\left(\mathrm{TM}_{010}\right)$ mode, which is expressed as

$$
\begin{aligned}
E_{z}^{010} & =e^{i\left(\omega t+\varphi_{0}\right)} E_{0} \cos (k z) J_{0}\left(k_{c} r\right) \\
& \approx e^{i\left(\omega t+\varphi_{0}\right)} E_{0} \cos (k z)\left(1-\frac{k_{c}^{2}}{4} r^{2}\right) \\
& =e^{i\left(\omega t+\varphi_{0}\right)} E_{0} \cos (k z)\left[1-\frac{k_{c}^{2}}{4}\left(x^{2}+y^{2}\right)\right] .
\end{aligned}
$$

If we define time $t$ to be zero when the beam centroid passes the cell center $(z=0)$, then $t=z / c$, and

$$
\text { beam energy }=e \int_{-L / 2}^{L / 2} \operatorname{Re}\left(E_{z}^{010}\right) d z=\frac{1}{2} e E_{0} L \cos \bar{\varphi}_{0} \text {, }
$$

where $\bar{\varphi}_{0}$ is the ensemble average of $\varphi_{0}$ along the electron distribution in the bunch. All electrons will see a different phase of rf field. We need to use average phase value to calculate the beam energy in Eq. (4). When $\bar{\varphi}_{0}$ is zero, the acceleration in the cell is maximized.

The normalized transverse monopole kick for a single electron can be calculated by the Panofsky-Wenzel theorem as

$$
\begin{aligned}
p_{n, \perp}^{010} & =\frac{1}{m c} \operatorname{Re}\left(\frac{e}{\omega} \int_{-L / 2}^{L / 2} i \nabla_{\perp} E_{z}^{010} d z\right) \\
& =\frac{k_{c}^{2}}{2} \alpha L \sin \varphi_{0}(x \hat{x}-y \hat{y}),
\end{aligned}
$$

where $\alpha=\left(e E_{0}\right) /\left(2 m c^{2} k\right)$ is the normalized $\mathrm{rf}$ field strength [1].

The normalized rms emittance growth is calculated by

$\varepsilon_{n, x}$

$=\sqrt{\left\langle\left(p_{n, x}-\left\langle p_{n, x}\right\rangle\right)^{2}\right\rangle\left\langle(x-\langle x\rangle)^{2}\right\rangle-\left\langle\left(p_{n, x}-\left\langle p_{n, x}\right\rangle\right)(x-\langle x\rangle)\right\rangle^{2}}$,

where the angle brackets mean statistical average. For the $y$ emittance $\varepsilon_{n, y}$, we can use same definition given in Eq. (6) with exchange of $x$ to $y$.

Thus, emittance growth due to monopole field is

$\varepsilon_{n, x}^{010}=\frac{k_{c}^{2}}{2} \alpha L \cos \bar{\varphi}_{0} \sigma_{x}^{2} \sigma_{\varphi} \quad \varepsilon_{n, y}^{010}=\frac{k_{c}^{2}}{2} \alpha L \cos \bar{\varphi}_{0} \sigma_{y}^{2} \sigma_{\varphi}$,

where $\sigma_{x}$ and $\sigma_{\varphi}$ are the rms beam size and the rms beam length (in the rf phase) averaged over the full cell region, respectively. Because the beam size and bunch length are changed in the full cell region, we need to average them in Eq. (7). In the above equation, we assume that the beam offset $\langle x\rangle$ and $\langle y\rangle$ in the full cell region are equal to zero. This assumption will be used for all calculation in next equations.

The dipole $\left(\mathrm{TM}_{110}\right)$ mode is

$$
\begin{aligned}
E_{z}^{110} & =e^{i\left(\omega t+\varphi_{0}\right)} E_{1} \cos (k z) J_{1}\left(k_{c} r\right) \cos \left(\theta-\frac{\pi}{2}\right) \\
& \approx e^{i\left(\omega t+\varphi_{0}\right)} E_{1} \cos (k z) \frac{k_{c} r}{2} \sin (\theta) \\
& =e^{i\left(\omega t+\varphi_{0}\right)} a_{1} E_{0} \cos (k z) r \sin (\theta) \\
& =e^{i\left(\omega t+\varphi_{0}\right)} a_{1} E_{0} \cos (k z) y,
\end{aligned}
$$

where $a_{1}$ is a parameter characterizing the relative strength of the dipole field to the monopole field.

Then the normalized transverse dipole kick for a single electron is 


$$
p_{n, \perp}^{110}=\frac{1}{m c} \operatorname{Re}\left(\frac{e}{\omega} \int_{-L / 2}^{L / 2} i \nabla_{\perp} E_{z}^{110} d z\right)=-a_{1} \alpha L \sin \varphi_{0} \hat{y}
$$

It shows that the dipole field kicks the beam only in the biggest port direction. If the beam passes the cell with maximum acceleration (i.e., $\bar{\varphi}_{0}=0$ ), there will be no net momentum kick from the dipole field.

The normalized emittance growth due to the dipole kick is calculated by Eq. (6) as

$$
\varepsilon_{n, y}^{110}=a_{1} \alpha L \cos \bar{\varphi}_{0} \sigma_{y} \sigma_{\varphi} .
$$

Similarly, the quadrupole $\left(\mathrm{TM}_{210}\right)$ mode is

$$
\begin{aligned}
E_{z}^{210} & =e^{i\left(\omega t+\varphi_{0}\right)} E_{2} \cos (k z) J_{2}\left(k_{c} r\right) \cos \left[2\left(\theta-\frac{\pi}{2}\right)\right] \\
& \approx-e^{i\left(\omega t+\varphi_{0}\right)} E_{2} \cos (k z) \frac{k_{c}^{2}}{8} r^{2}\left(\cos ^{2} \theta-\sin ^{2} \theta\right) \\
& =-e^{i\left(\omega t+\varphi_{0}\right)} a_{2} E_{0} \cos (k z)\left(x^{2}-y^{2}\right),
\end{aligned}
$$

where $a_{2}$ is a parameter characterizing the relative strength of the quadrupole field to the monopole field.

The normalized transverse quadrupole kick is

$$
\begin{aligned}
p_{n, \perp}^{210} & =\frac{1}{m c} \operatorname{Re}\left(\frac{e}{\omega} \int_{-L / 2}^{L / 2} i \nabla_{\perp} E_{z}^{210} d z\right) \\
& =2 a_{2} \alpha L \sin \varphi_{0}(x \hat{x}-y \hat{y}) .
\end{aligned}
$$

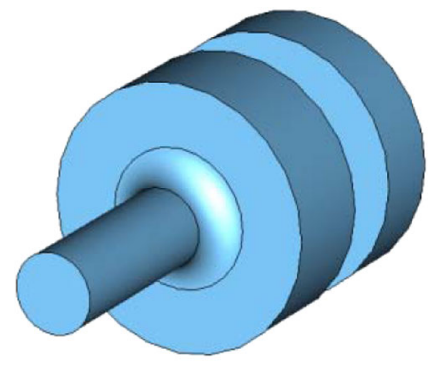

(a)

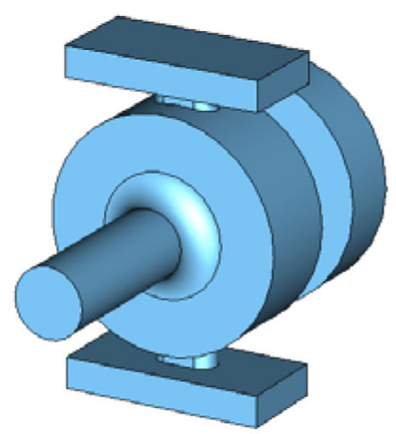

(c)

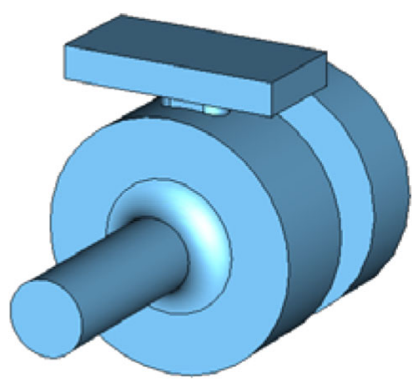

(b)

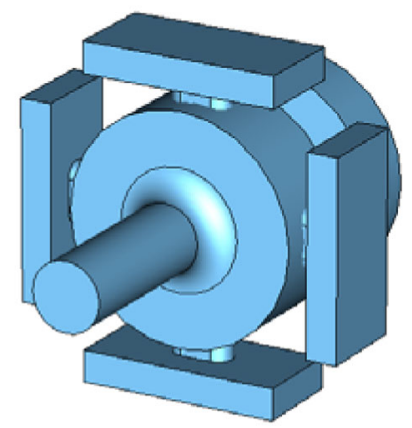

(d)
FIG. 1. Simulation models of the rf gun with (a) zero hole, (b) one hole, (c) two holes, and (d) four holes in the full cell part.
The ensemble average of the quadrupole kick $\left\langle p_{n, \perp}^{210}\right\rangle$ is zero. Thus, there will be no kicks from the quadrupole field. The normalized emittance growth due to the quadrupole kick is calculated as from Eq. (6) using Eq. (12),

$\varepsilon_{n, x}^{210}=2 a_{2} \alpha L \cos \bar{\varphi}_{0} \sigma_{x}^{2} \sigma_{\varphi} \quad \varepsilon_{n, y}^{210}=2 a_{2} \alpha L \cos \bar{\varphi}_{0} \sigma_{y}^{2} \sigma_{\varphi}$.

For the octopole $\left(\mathrm{TM}_{410}\right)$ mode, the accelerating electric field is given by

$$
\begin{aligned}
E_{z}^{410} & =e^{i\left(\omega t+\varphi_{0}\right)} E_{4} \cos (k z) J_{4}\left(k_{c} r\right) \cos \left[4\left(\theta-\frac{\pi}{2}\right)\right] \\
& \approx e^{i\left(\omega t+\varphi_{0}\right)} E_{4} \cos (k z) \frac{1}{4 !}\left(\frac{k_{c} r}{2}\right)^{4} \cos 4 \theta \\
& =e^{i\left(\omega t+\varphi_{0}\right)} a_{4} E_{0} \cos (k z)\left(x^{4}-6 x^{2} y^{2}+y^{4}\right),
\end{aligned}
$$

where $a_{4}$ is a parameter characterizing the relative strength of the octopole field to the monopole field.

The normalized transverse octopole kick is

$$
\begin{aligned}
p_{n, \perp}^{410} & =\frac{1}{m c} \operatorname{Re}\left(\frac{e}{\omega} \int_{-L / 2}^{L / 2} i \nabla_{\perp} E_{z}^{410} d z\right) \\
& =-4 a_{2} \alpha L \sin \varphi_{0}\left[\left(x^{3}-3 x y^{2}\right) \hat{x}-\left(y^{3}-3 x^{2} y\right) \hat{y}\right] .
\end{aligned}
$$

If the multipole field is weak enough so that the correlation between $x$ and $y$ is negligible, and the beam is still symmetric, then $\left\langle x^{2}\right\rangle=\left\langle y^{2}\right\rangle,\left\langle x^{4}\right\rangle=\left\langle y^{4}\right\rangle$, and the emittance induced by the octopole field is

$$
\begin{aligned}
& \varepsilon_{n, x}^{410}=4 \sqrt{6} a_{4} \alpha L \sigma_{x}^{4} \sqrt{\sin ^{2} \bar{\varphi}_{0}+\sigma_{\varphi}^{2} \cos ^{2} \bar{\varphi}_{0}} \\
& \varepsilon_{n, y}^{410}=4 \sqrt{6} a_{4} \alpha L \sigma_{y}^{4} \sqrt{\sin ^{2} \bar{\varphi}_{0}+\sigma_{\varphi}^{2} \cos ^{2} \bar{\varphi}_{0}} .
\end{aligned}
$$

As we can see later in Sec. IV, the octopole field effect could be neglected. If we consider only monopole, dipole, and quadrupole effect, the rf field could be approximated as
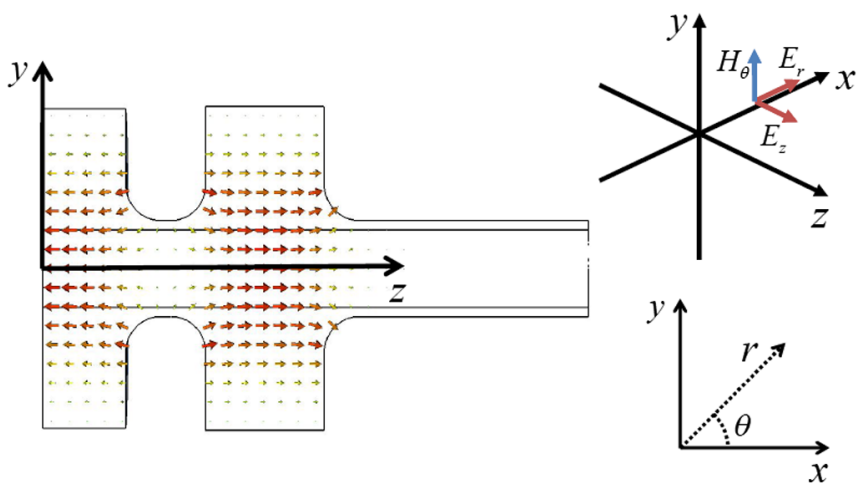

FIG. 2. Electromagnetic field distribution in the rf gun. The phase of the field is 0 degree. 


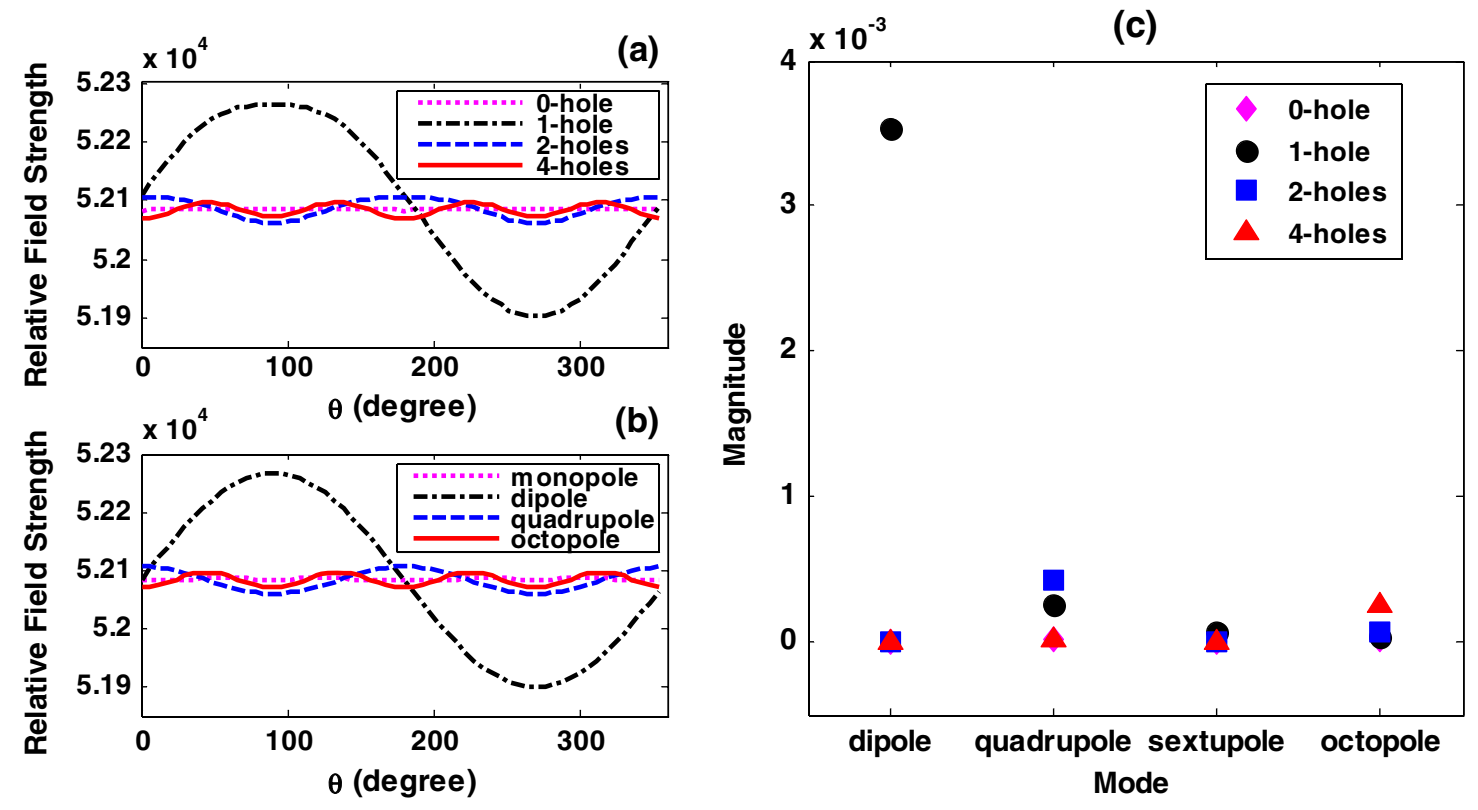

FIG. 3. Field distribution along the $\theta$ direction. (a) The rf field $E_{r}$ distribution along the $\theta$ direction for the models shown in Fig. 1 at $z=61 \mathrm{~mm}$ (middle of the full cell), $r=9.5 \mathrm{~mm}$. (b) Distribution of the artificial $\mathrm{rf}$ field, which is the combination of the monopole and the dominant component of each model. (c) Fourier coefficient of $E_{r}$ of 1.6 cell models with a different number of holes.

$$
\begin{aligned}
E_{z} \approx & E_{z}^{010}+E_{z}^{110}+E_{z}^{210} \\
= & e^{i\left(\omega t+\varphi_{0}\right)} \cos (k z) E_{0}\left[1+a_{1} y-\left(a_{2}+\frac{k_{c}^{2}}{4}\right) x^{2}\right. \\
& \left.-\left(-a_{2}+\frac{k_{c}^{2}}{4}\right) y^{2}\right] .
\end{aligned}
$$

The total normalized transverse rf field kick is

$$
\begin{aligned}
& p_{n, x}=\left(2 a_{2}+\frac{k_{c}^{2}}{2}\right) x \alpha L \sin \varphi_{0} \hat{x} \\
& p_{n, y}=-\left[a_{1}+\left(2 a_{2}-\frac{k_{c}^{2}}{2}\right) y\right] \alpha L \sin \varphi_{0} \hat{y} .
\end{aligned}
$$

The total emittance growth due to the rf effect calculated by Eq. (6) is

$$
\begin{aligned}
\varepsilon_{n, x}^{010+110+210} & =\left(\frac{k_{c}^{2}}{2}+2 a_{2}\right) \alpha L \cos \bar{\varphi}_{0} \sigma_{x}^{2} \sigma_{\varphi}=\varepsilon_{n, x}^{010}+\varepsilon_{n, x}^{210} \\
\varepsilon_{n, y}^{010+110+210} & =\alpha L \cos \bar{\varphi}_{0} \sigma_{y} \sigma_{\varphi} \sqrt{\left(\frac{k_{c}^{2}}{2}-2 a_{2}\right)^{2} \sigma_{y}^{2}+a_{1}^{2}} \\
& =\sqrt{\left(\varepsilon_{n, y}^{010}-\varepsilon_{n, y}^{210}\right)^{2}+\left(\varepsilon_{n, y}^{110}\right)^{2}} .
\end{aligned}
$$

If the dipole field is the dominant multipole field ( $\mathrm{rf}$ gun with one hole), the quadrupole field can be neglected, then

$$
\begin{aligned}
\varepsilon_{n, x}^{010+110} & =\frac{k_{c}^{2}}{2} \alpha L \cos \bar{\varphi}_{0} \sigma_{x}^{2} \sigma_{\varphi}=\varepsilon_{n, x}^{010} \\
\varepsilon_{n, y}^{010+110} & =\alpha L \cos \bar{\varphi}_{0} \sigma_{y} \sigma_{\varphi} \sqrt{\left(\frac{k_{c}^{2}}{2} \sigma_{y}\right)^{2}+a_{1}^{2}} \\
& =\sqrt{\left(\varepsilon_{n, y}^{010}\right)^{2}+\left(\varepsilon_{n, y}^{110}\right)^{2}} .
\end{aligned}
$$

TABLE I. Fourier coefficient $\left(a_{n} r^{n}\right)$ of $E_{z}$ for the models in Fig. 1. The dominant mode in each case is boldfaced. These values are computed at the position $z=61 \mathrm{~mm}$, which is the center of the full cell, and the radius $r_{0}=9.5 \mathrm{~mm}$.

\begin{tabular}{lcccc}
\hline \hline Mode & 0 hole & 1 hole & 2 holes & 4 holes \\
\hline$a_{0} / a_{0}$, monopole & $\mathbf{1}$ & 1 & 1 & 1 \\
$a_{1} r_{0} / a_{0}$, dipole & $3.75 \times 10^{-22}$ & $\mathbf{4 . 9 2} \times \mathbf{1 0}^{-\mathbf{3}}$ & $3.93 \times 10^{-20}$ & $5.06 \times 10^{-22}$ \\
$a_{2} r_{0}^{2} / a_{0}$, quadrupole & $9.34 \times 10^{-6}$ & $7.47 \times 10^{-4}$ & $\mathbf{1 . 5 1} \times \mathbf{1 0}^{-\mathbf{3}}$ & $1.42 \times 10^{-6}$ \\
$a_{3} r_{0}{ }^{3} / a_{0}$, sextupole & $1.28 \times 10^{-23}$ & $1.46 \times 10^{-4}$ & $6.85 \times 10^{-23}$ & $2.63 \times 10^{-23}$ \\
$a_{3} r_{0}{ }^{4} / a_{0}$, octopole & $3.41 \times 10^{-7}$ & $2.94 \times 10^{-5}$ & $5.97 \times 10^{-5}$ & $\mathbf{1 . 2 3} \times \mathbf{1 0}^{-4}$ \\
\hline \hline
\end{tabular}




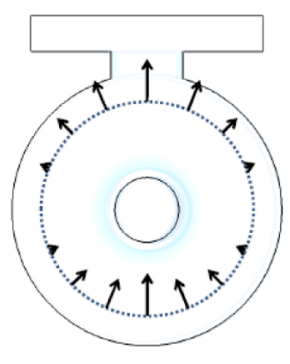

(a)

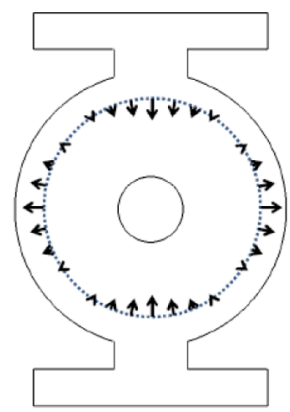

(b)

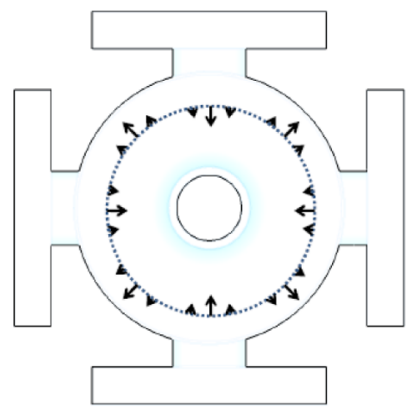

(c)

FIG. 4. Schematic drawing of each multipole mode in the rf gun. (a) Dipole mode. (b) Quadrupole mode. (c) Octopole mode.

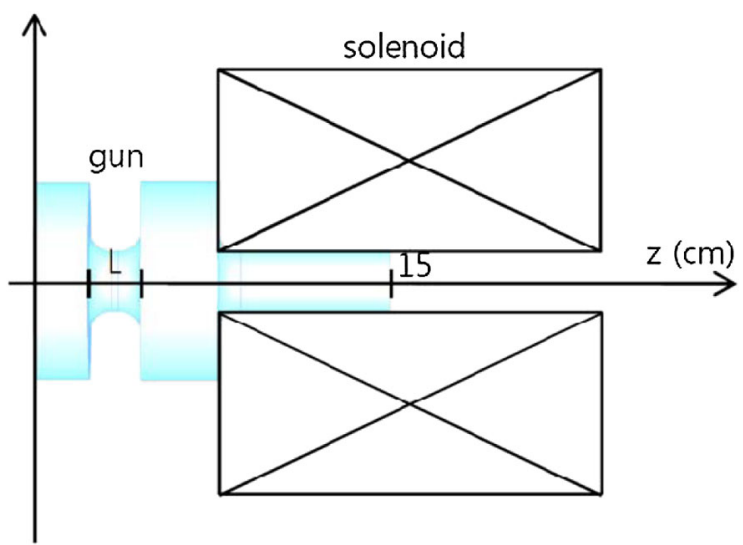

FIG. 5. Simulation layout. Gun and solenoid magnet.

As we can see in Eq. (20) there is no emittance growth in the $x$ axis for the dipole mode case. For the $y$ axis, there will be emittance growth in the quadrature sum between the monopole mode emittance and the dipole mode emittance.

If the quadrupole field is the dominant multipole field ( $\mathrm{rf}$ gun with two holes), the dipole field can be neglected, then

TABLE II. Parameters used in PARMELA simulation. These values are computed at the position $z=61 \mathrm{~mm}$, which is the center of the full cell, and the radius $r_{0}=9.5 \mathrm{~mm} . \sigma_{1 \text {-hole }}$, $\sigma_{2 \text {-holes }}$ is the rms beam size of the one-hole model and the two-holes model at the same longitudinal position $(z=61 \mathrm{~mm})$, respectively. $\varphi_{0}$ is the phase of the rf field. Initial beam sizes are edge values. The input beam has a beer can shape. There is no initial thermal emittance.

\begin{tabular}{ll}
\hline \hline Number of particles & 100000 \\
Total charge & $0 / 0.1 / 1 \mathrm{nC}$ \\
UV laser pulse length (FWHM) & $10 \mathrm{ps}$ \\
UV laser radius & $1.2 \mathrm{~mm}$ \\
Initial kinetic energy at the cathode & $1 \mathrm{eV}$ \\
Peak $E_{0}$ at cathode & $120 \mathrm{MV} / \mathrm{m}$ \\
Length of the rf gun & $15 \mathrm{~cm}$ \\
Initial laser-rf phase & $32 \mathrm{degree}$ \\
\hline \hline
\end{tabular}

$$
\begin{aligned}
& \varepsilon_{n, x}^{010+210}=\left(\frac{k_{c}^{2}}{2}+2 a_{2}\right) \alpha L \cos \bar{\varphi}_{0} \sigma_{x}^{2} \sigma_{\varphi}=\varepsilon_{n, x}^{010}+\varepsilon_{n, x}^{210} \\
& \varepsilon_{n, y}^{010+210}=\left(\frac{k_{c}^{2}}{2}-2 a_{2}\right) \alpha L \cos \bar{\varphi}_{0} \sigma_{y}^{2} \sigma_{\varphi}=\varepsilon_{n, y}^{010}-\varepsilon_{n, y}^{210} .
\end{aligned}
$$

According to Eq. (21), when the quadrupole field is the dominant one, it seems to be reasonable to consider the emittance as the linear sum or subtraction of contributions between the quadrupole mode and the monopole mode rather than the quadrature sum as done in the dipole

(a)

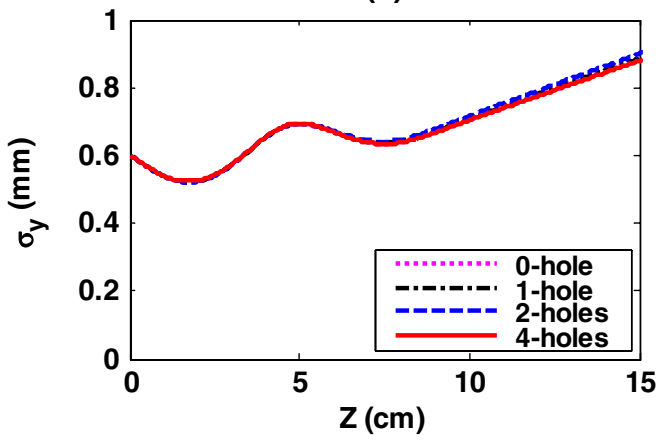

(b)

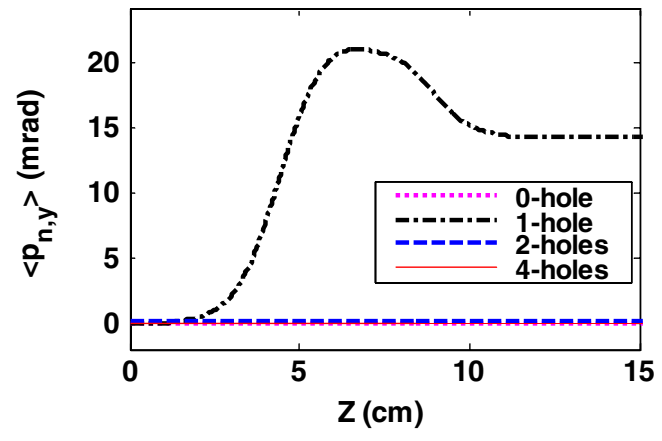

FIG. 6. (a) The rms beam size and (b) normalized transverse momentum of the $0 \mathrm{nC}$ charged beam in the vertical direction without solenoid field drawn by PARGRAF. It is shown that there exists a 14 mrad normalized transverse momentum in the vertical (y) direction. 
(a)

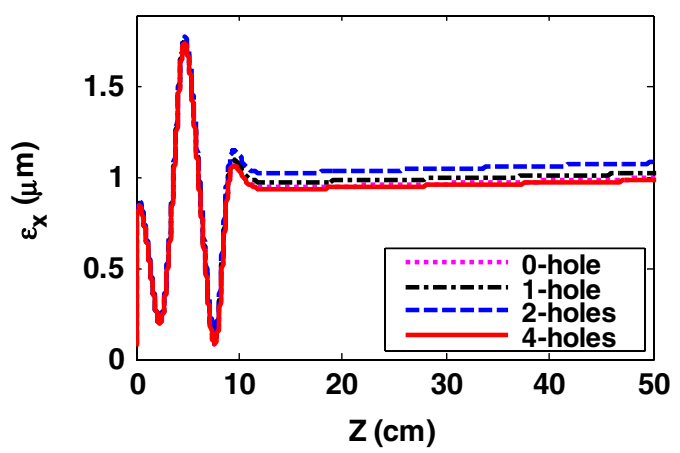

(c)

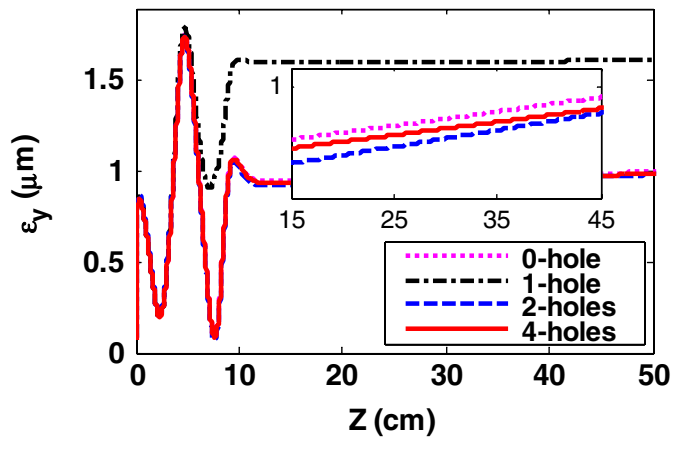

(b)

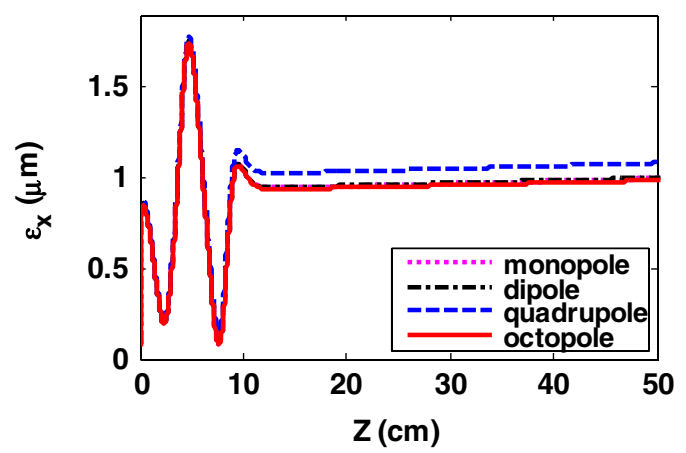

(d)

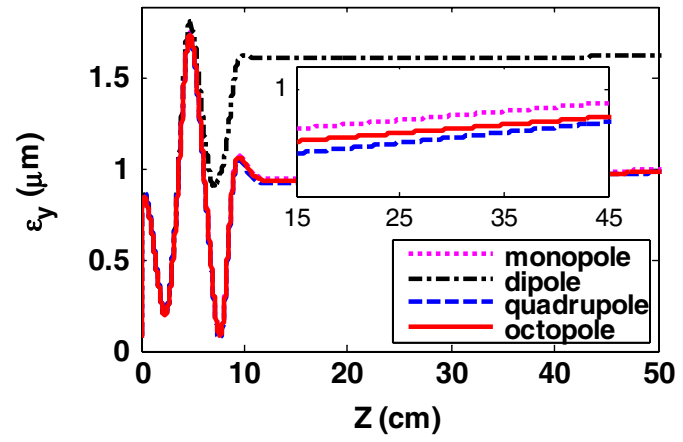

FIG. 7. Simulation results of rms emittance in both directions without solenoid field for the beam charge of $0 \mathrm{nC}$. (a) Emittance evolutions in the horizontal $(x)$ direction for each model. (b) Emittance evolutions from the dominant multipole field from each model in the horizontal $(x)$ direction. (c) Emittance evolutions in the vertical $(y)$ direction for each model. (d) Emittance evolutions from the dominant multipole field from each model in the vertical $(y)$ direction.

mode case. This is why in the gun model with two holes, which will be explained in Sec. III, emittance seems to be different in $x$ and $y$ directions, i.e., emittance in the $x$ direction is bigger than the monopole mode and emittance in the $y$ direction is smaller than the monopole mode.

The above derivations consider pure rf effects without space-charge effect and solenoid-focusing effect. Since the beam rotates in a solenoid field, the multipole field effects will be coupled to both $x$ and $y$ directions. For example, the dipole kick is expected to affect the beam in both directions, which will be shown in Sec. IV B.

TABLE III. Parameters used in numerical calculation of the beam offset and the emittance growth.

\begin{tabular}{ll}
\hline \hline$E_{0}(\mathrm{MV} / \mathrm{m})$ & 120 \\
$f(\mathrm{MHz})$ & 2856 \\
$a_{1}$ at $r_{0}$ & 0.517 \\
$a_{2}$ at $r_{0}$ & 16.73 \\
$r_{0}(\mathrm{~mm})$ & 9.5 \\
$L(\mathrm{~mm})$ & 51.562 \\
$\bar{\varphi}_{0}($ degree $)$ & 8 \\
$k E(\mathrm{MeV})$ & 5.6 \\
$\sigma_{1 \text {-hole, } x}(\mathrm{~mm})$ & 0.6573 \\
$\sigma_{2 \text {-holes, } y}(\mathrm{~mm})$ & 0.6611 \\
$\bar{\sigma}_{z}(\mathrm{ps})$ & 2.1 \\
\hline \hline
\end{tabular}

\section{SIMULATION MODELS AND RF FIELD MANIPULATION}

\section{A. Simulation models and axes definition}

We have performed a simulation study to understand beam dynamics concerning the field asymmetry in an $\mathrm{rf}$ gun. In Appendix A, the detail specification of the reference rf gun used in this study is given. A 1.6-cell $\mathrm{rf}$ gun model without any coupling hole was designed. Then, we made additional holes to the side of the full cell. Finally, four kinds of gun models were designed, which has no hole, one hole, two holes, and four holes, in turn. These four kinds of rf gun models are shown in Fig. 1.

It is important to note that we studied the effect of internal field distribution perturbed by the change of external boundary of the gun cavity in this study. Any port in the model can be considered as ports for power feed. There can be a power flow effect on the emittance growth. This topic is not covered in this study. However, in the practical application, if the dual feed scheme is used in the four-holes gun, we can expect that the power flow effect can be ignored [19].

An electromagnetic field distribution in the rf gun cavity is shown in Fig. 2. We defined the central axis of the cavity as the $z$ axis having the origin located at the cathode. The vertical axis corresponding to the waveguide port direction 
is denoted as $y$ and the horizontal axis is denoted as $x$. The azimuthal angle $\theta$ is defined in the $x-y$ plane. The field distribution shown in Fig. 2 is the plot of the electromagnetic field in the rf gun when the accelerating phase is 0 degree. Here, the direction of the radial electric field $\left(E_{r}\right)$ is outward from the $z$ axis and that of the azimuthal magnetic field $\left(B_{\theta}\right)$ is counterclockwise. This distribution will oscillate as the resonance frequency of the rf gun.

\section{B. rf field manipulation}

Using the fast Fourier transform (FFT) algorithm, all the required information is obtained, such as magnitudes and phases of all multipole modes. Using this information, an artificial rf field can be created by adding or subtracting certain modes. In our study, the FFT was done with respect to the spatial coordinate (azimuthal angle $\theta$ in the cavity), not time coordinate, and a detail explanation for the FFT process is given in Appendix B 1.

We obtained four kinds of 3D rf field maps for the models shown in Fig. 1. By manipulating the modes in the field distributions, we also made four other artificial kinds of 3D rf field maps, viz. monopole only, monopole plus dipole, monopole plus quadrupole, and monopole plus octopole. The amplitudes of the four artificial field distributions were obtained from the models with zero hole, one hole, two holes, and four holes. By using these artificial fields, the pure effect of the dominant mode on the emittance growth can be discriminated from the whole effect of the original rf field distribution in each model.

Field distributions along the azimuthal direction $\theta$ for the PARMELA input are shown in Figs. 3(a) and 3(b). These values are computed at the position $z=61 \mathrm{~mm}$, which is the center of the full cell, and the radius $r_{0}=9.5 \mathrm{~mm}$. Figure 3(a) shows the $E_{r}$ distribution of each model with a different number of holes as drawn in Fig. 1. The distributions of a dominant multipole mode with the monopole mode in each model are shown in Fig. 3(b). The dipole field in Fig. 3(b) is the exact cosine function, though the field of the one-hole model in Fig. 3(a) is distorted by the effect of higher modes. Figure 3(c) shows the amplitudes of all Fourier modes for each model. For the one-hole model, the dipole mode is an absolutely dominant mode. In the case of a two-holes model, the amplitude of the quadrupole mode is larger than that of the dipole mode. For the four-holes model, the dipole mode and quadrupole mode are insignificant, and the octopole mode is the largest. In summary, the dipole mode, the quadrupole mode, and the octopole mode are the largest modes in the onehole model, the two-holes model, and the four-holes model, respectively. Note that all amplitudes are normalized by the amplitude of the monopole mode. For the theoretical calculation, we need to know also the normalized amplitudes of each mode (i.e., the Fourier coefficient of the longitudinal field $E_{z}$ ), which are listed also in Table I.
Figure 4 shows the schematic drawings of the transverse electric field distribution of each multipole mode, which gives us an intuition for the relation between each mode and the emittance growth. In Fig. 4(a), the dipole mode gives the electron beam a kick in $y$ direction, but it has no effect in the $x$ direction. Meanwhile, the quadrupole mode applies a kick inward in the $y$ direction and outward in the $x$ direction as shown in Fig. 4(b). In Fig. 4(c), the octopole mode is drawn, and it applies a kick outward in the $x$ and $y$ directions and inward in a direction that is rotated 45 degrees with respect to the $x$ and $y$ axes. Note that the relative magnitude of the dipole mode with respect to the monopole mode at the position of $9.5 \mathrm{~mm}$ radius is of the order of $10^{-3}$. Therefore, the fields shown in Fig. 4 bear no relation to the actual magnitude of the rf field.

\section{SIMULATION RESULTS}

A PARMELA simulation with an rf gun was performed with the 3D rf field maps, which were constructed using the method mentioned in Sec. III. The simulation configuration is drawn in Fig. 5, and the simulation parameters are listed in Table II. Initial beam sizes in Table II are edge values. The input beam has a beer can shape, i.e., uniformly distributed both transversely and longitudinally. Initial thermal emittance is set to be zero in simulation. More details of the simulation procedure are given in

(a)

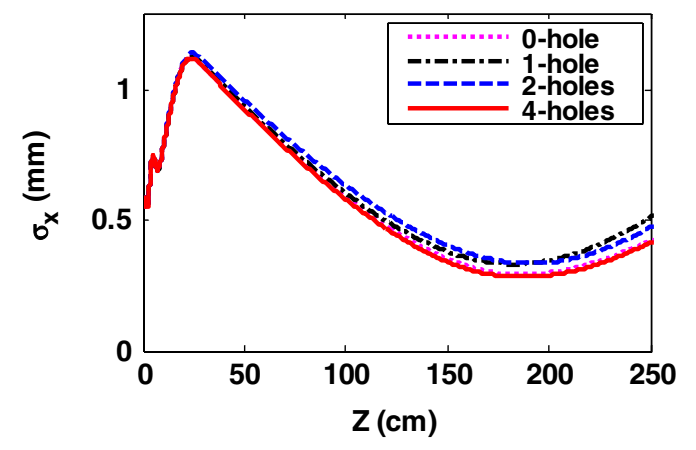

(b)

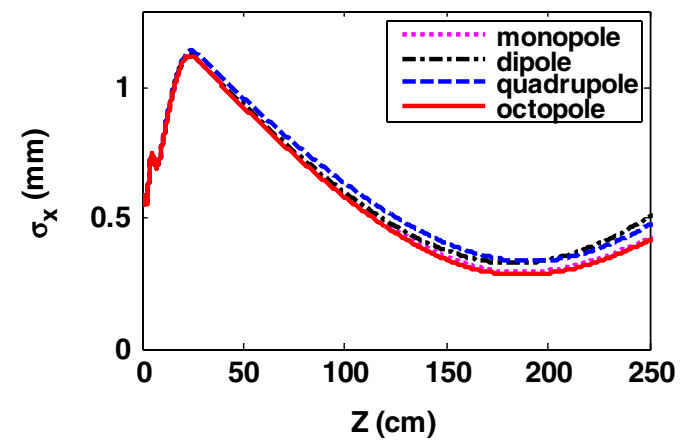

FIG. 8. $X$ rms beam size of the rf gun with solenoid field for the beam charge $0.1 \mathrm{nC}$ (a) with the four rf gun models and (b) with four artificial field maps. 
Appendix B 2. To understand beam dynamics clearly, we simulated the electron beam evolution with the PARMELA code using three kinds of beam charges. First, the beam charge of $0 \mathrm{nC}$ was studied without solenoid field to understand the effect of the rf field distribution to the emittance growth. The other beams with charges of 0.1 and $1 \mathrm{nC}$ were also studied with solenoid field.

\section{A. 0 nC charge without solenoid field}

The simulation of the $0 \mathrm{nC}$ charge case has been carried out without solenoid field. Evolutions of the rms beam size in the four models are shown in Fig. 6(a). They show similar behaviors. Evolution of the average transverse momentum of electron beam, $\left\langle p_{n, y}\right\rangle$ in the one-hole case is shown with a black dash-dotted line in Fig. 6(b). The effect of the transverse kick due to dipole field is clearly shown in Fig. 6(b). The normalized transverse momentum is $14.2 \mathrm{mrad}$ at the exit of the cavity. This transverse kick due to the dipole field in the vertical $(y)$ direction can be calculated analytically by using Eq. (9) in Sec. II. The electron will be kicked in the vertical $(y)$ direction by $7.25 \mathrm{mrad}$ from Eq. (9), which is about half of the simulation result. The difference between the result of simulation and calculation is conjectured to be a result from the difference of the models we considered in calculation and simulation. We used a 1.6-cell model in the simulation. But the cavity model considered in the theory part, Sec. II, is a single cell model. We cannot apply the PanofskyWenzel theorem to the half cell case since the velocity of the beam is much smaller than $c$ in the half cell. In the simulation, there is a transverse kick about $4 \mathrm{mrad}$ from the half cell which is shown in Fig. 6(b). Thus, there is roughly a $10 \mathrm{mrad}$ kick in the full cell in the simulation. This value is reasonable to be compared with the theoretical prediction, 7.25 mrad.

According to Eqs. (12) and (15), in the two-holes model and the four-holes model, there will be no average kicks, viz. $\left\langle p_{n, x}\right\rangle=\left\langle p_{n, y}\right\rangle=0$. It is also clearly shown in the simulation results in Fig. 6(b). There is no dipole kick at all for the rf guns with two and four holes. All parameters used in the calculation here are shown in Table III in which $r_{0}$ is the radius where the FFT analysis of $\mathrm{rf}$ field is implemented, $L$ is the length of the full cell, $\sigma_{1 \text {-hole, } x}$, $\sigma_{2 \text {-hole, } x}$ are the averaged $\mathrm{rms}$ beam size of the one-hole model and the two-holes model in the full cell region, respectively, and $\bar{\varphi}_{0}$ is the ensemble averaged rf phase with the electron distribution in the beam.

Figure 7(a) shows the emittance evolutions in the horizontal $(x)$ direction for each model. In Fig. 7(b), the $x$-emittance evolutions from the dominant multipole field (a)

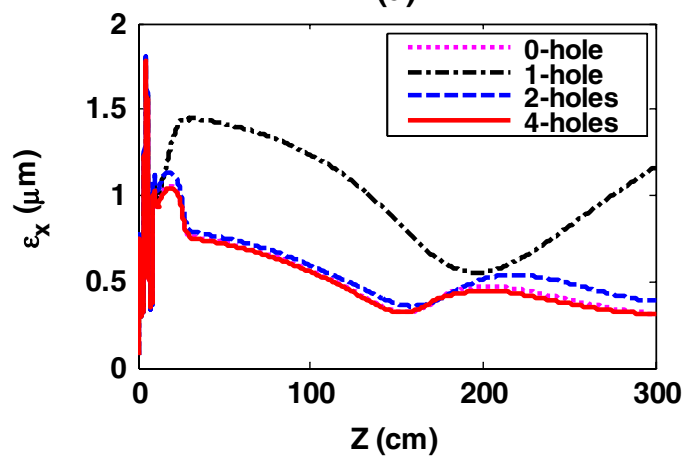

(c)

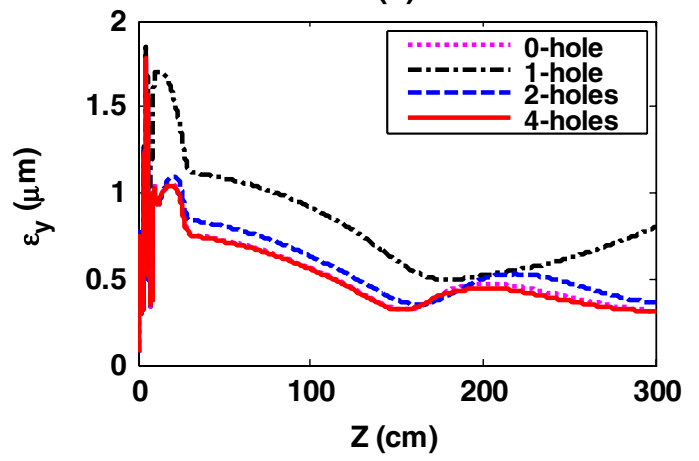

(b)

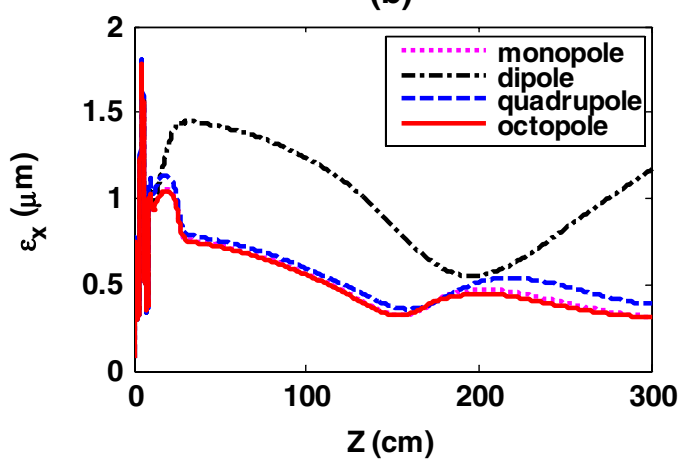

(d)

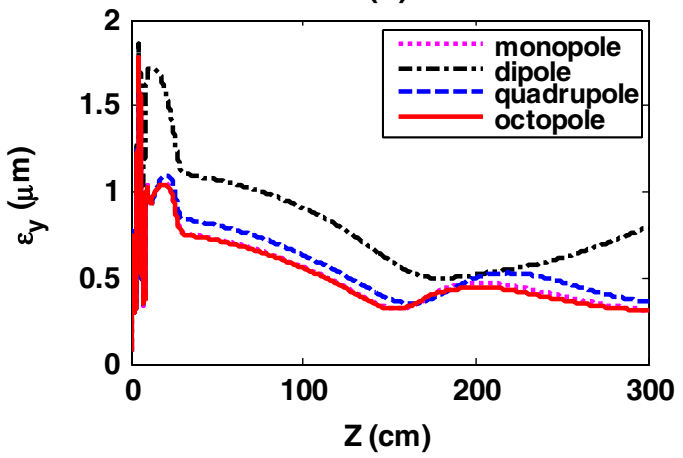

FIG. 9. Simulation results of rms emittance in both directions without solenoid field for the beam charge of $0.1 \mathrm{nC}$. (a) Emittance evolutions in the horizontal $(x)$ direction for each model. (b) Emittance evolutions from the dominant multipole field from each model in the horizontal $(x)$ direction. (c) Emittance evolutions in the vertical (y) direction for each model. (d) Emittance evolutions from the dominant multipole field from each model in the vertical $(y)$ direction. 
from each model in the horizontal $(x)$ direction are shown. Note that the emittance for the monopole field is not zero but $0.948 \mu \mathrm{m}$. From Eq. (7), the theoretical emittances with the monopole field are calculated as

$$
\varepsilon_{n, x}^{010} \approx \varepsilon_{n, y}^{010}=\frac{k_{c}^{2}}{2} \alpha L \cos \bar{\varphi}_{0} \sigma_{x}^{2} \sigma_{\varphi}=2.624(\mu \mathrm{m}) .
$$

The theoretical predictions are higher than the simulated one. This difference may come from the fact that the model in Sec. II is a single cell, while that in simulation is a 1.6 cell. The emittance growth due to monopole mode can be also calculated from Eq. (35) of Ref. [1] and the result is $0.052 \mu \mathrm{m}$ [20]. These differences in two theoretical predictions and simulation are not fully understood yet.

The emittance in the horizontal $(x)$ direction was almost unaffected by all models except the two-holes model. Note that all the emittances in this study are projected emittances and the slice emittances also show similar behaviors which is not shown in this study.

The simulation results of the emittance for each multipole mode are shown in Fig. 7(b). Only the quadrupole field (blue dashed line) case shows higher emittance than the other cases. By comparing the simulation results shown in Figs. 7(a) and 7(b), we can conclude that the quadrupole mode is the dominant mode in the two-holes model.

Figure 7(c) shows the emittance evolution in the vertical $(y)$ direction for each model. The results for each multipole field are shown in Fig. 7(d). In the case of the vertical direction, the emittance from the one-hole model in Fig. 7(c) and the dipole mode in Fig. 7(d) were significantly increased as compared to the other cases. Note that the emittance in the two-holes model is lower than the emittance in the zero-hole model. This behavior can be seen in the inset of Fig. 7(c) which is added for clarity. It is also observed in the quadrupole field case in the inset of Fig. 7(d). By comparing the simulation results shown in Figs. 7(c) and 7(d), we can conclude that the dipole (quadrupole) mode is the dominant mode in the onehole (two-hole) model.

According to Eq. (10) in Sec. II, emittance growth at the gun exit is calculated as $1.257 \mu \mathrm{m}$ for the dipole mode in the vertical $(y)$ direction. All parameters including the amplitudes of the multipole mode in each gun model used in the analytical calculation here are listed in Table III. In Fig. 7(d), the simulated emittance at the gun exit for the dipole mode case in the vertical ( $y$ ) direction is $\varepsilon_{n, y}^{010+110}=1.601 \mu \mathrm{m}$. Here the dipole mode case means monopole mode plus dipole mode. The emittance growth due to the dipole field is calculated from Eq. (20) as follows:

$$
\varepsilon_{n, y}^{110} \approx \sqrt{\left(\varepsilon_{n, y}^{010+110}\right)^{2}-\left(\varepsilon_{n, y}^{010}\right)^{2}}=1.290(\mu \mathrm{m}) .
$$

This value well matches with the theory prediction from Eq. (10).
TABLE IV. Minimum emittance for $0.1 \mathrm{nC}$ charge.

\begin{tabular}{lcccc}
\hline \hline Field & $z(\mathrm{~cm})$ & $\varepsilon_{x}(\mu \mathrm{m})$ & $z(\mathrm{~cm})$ & $\varepsilon_{y}(\mu \mathrm{m})$ \\
\hline 0 hole & 155 & 0.319 & 155 & 0.322 \\
1 hole & 200 & 0.513 & 181 & 0.497 \\
2 holes & 160 & 0.357 & 162 & 0.352 \\
4 holes & 154 & 0.319 & 154 & 0.319 \\
Dipole mode & 196 & 0.548 & 180 & 0.499 \\
Quadrupole mode & 162 & 0.361 & 163 & 0.352 \\
Octopole mode & 154 & 0.319 & 154 & 0.319 \\
\hline \hline
\end{tabular}

The simulated emittance at the gun exit for the quadrupole mode case in the horizontal $(x)$ direction in Fig. 7(b), $\varepsilon_{n, x}^{010+210}$ is $1.027 \mu \mathrm{m}$ and, in Fig. $7(\mathrm{~d}), \varepsilon_{n, y}^{010+210}$ is $0.924 \mu \mathrm{m}$ in the vertical $(y)$ direction. The quadrupole mode case also means monopole mode plus quadrupole mode. As mentioned in Sec. II, we can calculate the emittance of the two-holes model as the linear sum of the emittance of the monopole mode and the quadrupole mode as shown in Eq. (21) in Sec. II as follows:

$\varepsilon_{n, x}^{210}=\varepsilon_{n, x}^{010+210}-\varepsilon_{n, x}^{010}=1.027-0.947=0.080(\mu \mathrm{m})$

$\varepsilon_{n, y}^{210}=-\varepsilon_{n, y}^{010+210}+\varepsilon_{n, y}^{010}=-0.924+0.948=0.024(\mu \mathrm{m})$.

We can say that there are emittance growths of about $0.080 \mu \mathrm{m}$ in the horizontal $(x)$ direction and an emittance

(a)

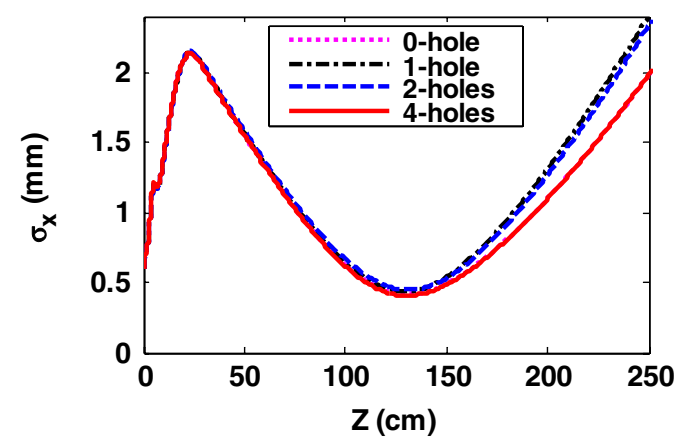

(b)

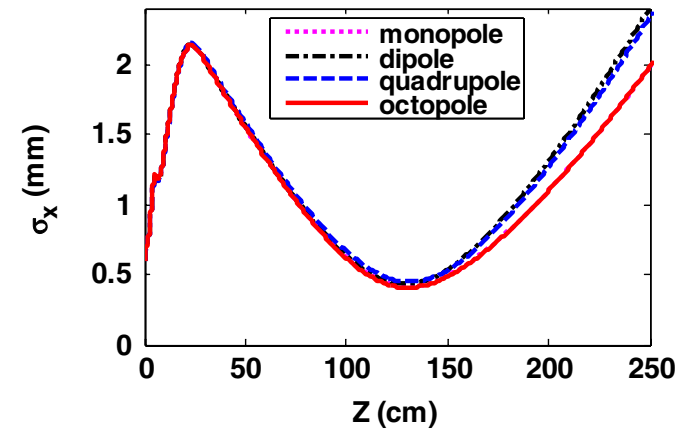

FIG. 10. $\quad X$ rms beam size of the rf gun with solenoid field for the beam charge $1 \mathrm{nC}$ (a) with the four rf gun models and (b) with four artificial field maps. 
decrease of about $0.024 \mu \mathrm{m}$ due to the quadrupole mode at the gun exit. According to Eq. (13) in Sec. II, emittance growth is predicted as $0.0536 \mu \mathrm{m}$ in the horizontal $(x)$ direction and emittance decrease is predicted as $0.0542 \mu \mathrm{m}$ in the vertical $(y)$ direction. The emittance growths in $x$ and $y$ directions are close to the theory predictions.

\section{B. 0.1 nC charge with solenoid field}

For the successful operation of the fourth generation light source, low-charge operations with beam charges as low as $0.1 \mathrm{nC}$ are also being studied [21]. To understand the beam dynamics on the low-charge operation, we performed a simulation using a beam charge of $0.1 \mathrm{nC}$. Figure 8(a) shows the results of the rms beam size in the horizontal $(x)$ direction of the four models. Figure 8(b) shows the rms beam sizes of each dominant mode of the four models. The rms beam sizes were not significantly affected by the field asymmetries. However, the evolutions of emittance show considerable differences in behaviors according to each model.

The results of the emittance evolution in the horizontal $(x)$ direction are shown in Fig. 9(a). The emittance of the one-hole model increased rapidly. The emittance evolu- tions with other models show double minimums [22]. At all longitudinal positions, the emittance of the one-hole model is the highest. The two-holes model shows the next higher value. The zero-hole model and the four-holes model do not show much difference, and their emittance values are the lowest. The emittance evolution with the dominant multipole mode in each model shows very similar behavior as shown in Fig. 9(b). The emittance evolutions in the vertical $(y)$ direction are shown in Figs. 9(c) and 9(d). The overall behaviors in the vertical direction are the same with the horizontal direction. A comparison of the figures shows that the dominant modes are the dipole, quadrupole, and octopole modes in the onehole, two-holes, and four-holes models, respectively. The values of the minimum emittance for each case in Fig. 9 are listed in Table IV.

\section{1 nC charge with solenoid field}

A high-beam charge operation scheme of $1 \mathrm{nC}$ was also considered in the fourth generation light source [10]. The emittance evolution with the beam charge of $1 \mathrm{nC}$ was also simulated in this study. The results of the rms beam size in the horizontal $(x)$ direction showed insignificant differences for all kinds of models as shown in Fig. 10. (a)

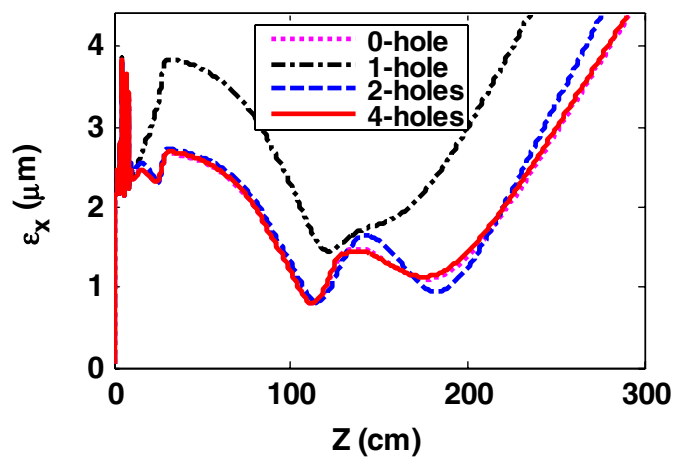

(c)

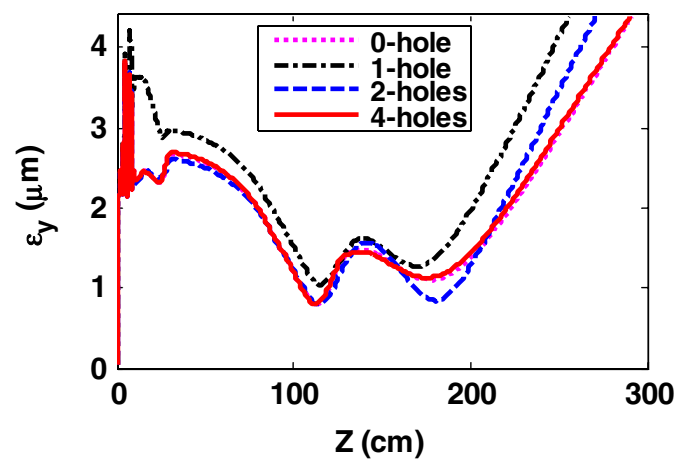

(b)

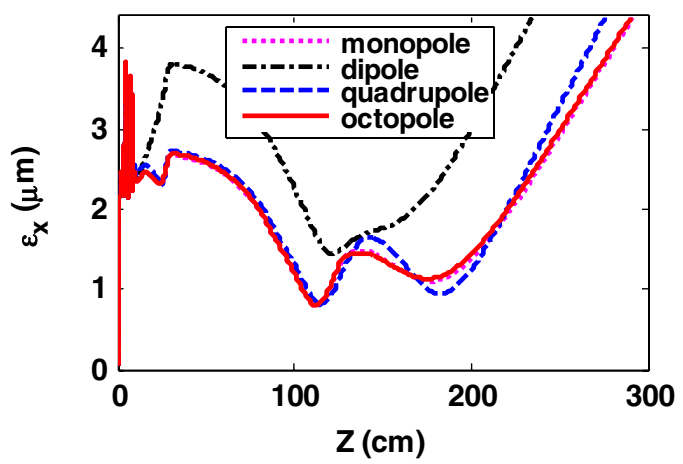

(d)

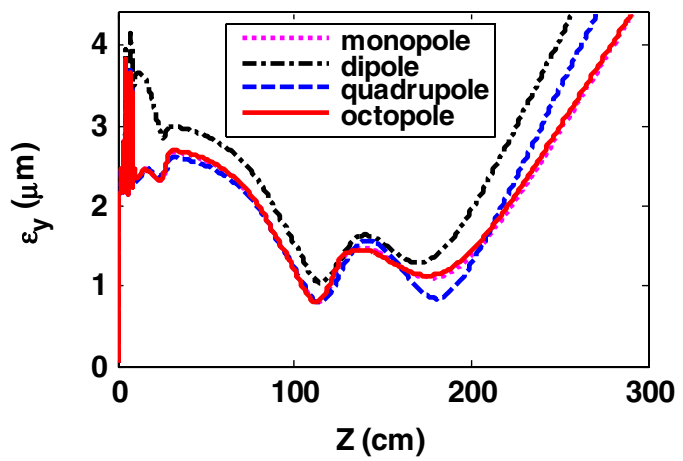

FIG. 11. Simulation results of rms emittance in both directions without solenoid field for the beam charge of $1 \mathrm{nC}$. (a) Emittance evolutions in the horizontal $(x)$ direction for each model. (b) Emittance evolutions from the dominant multipole field from each model in the horizontal $(x)$ direction. (c) Emittance evolutions in the vertical $(y)$ direction for each model. (d) Emittance evolutions from the dominant multipole field from each model in the vertical $(y)$ direction. 
TABLE V. Minimum emittance for $1 \mathrm{nC}$ charge.

\begin{tabular}{lcccc}
\hline \hline Field & $z(\mathrm{~cm})$ & $\varepsilon_{x}(\mu \mathrm{m})$ & $z(\mathrm{~cm})$ & $\varepsilon_{y}(\mu \mathrm{m})$ \\
\hline 0 hole & 113 & 0.799 & 113 & 0.798 \\
1 hole & 122 & 1.455 & 115 & 1.045 \\
2 holes & 115 & 0.814 & 114 & 0.797 \\
4 holes & 112 & 0.800 & 112 & 0.799 \\
Dipole mode & 122 & 1.450 & 115 & 1.046 \\
Quadrupole mode & 115 & 0.814 & 114 & 0.797 \\
Octopole mode & 112 & 0.800 & 112 & 0.799 \\
\hline \hline
\end{tabular}

The results of the emittance evolution in the horizontal $(x)$ direction are shown in Figs. 11(a) and 11(b). Double minimums in the emittance evolution are clearly seen for a beam charge of $1 \mathrm{nC}$ [22]. The emittance values for the one-hole model in Fig. 11(a) and the dipole mode in Fig. 11(b) show the highest values. The emittance evolutions in the vertical $(y)$ direction are shown in Figs. 11(c) and 11(d). Their overall behavior is similar to that in the horizontal $(x)$ direction. The emittance at the first minimum position in both directions is roughly $0.9 \mu \mathrm{m}$ with the exception of the results of the one-hole model in the horizontal $(x)$ direction. The minimum emittances in Fig. 11 are listed in Table V.

We noticed that the emittance evolution in the horizontal $(x)$ direction is much higher than the emittance in the vertical $(y)$ direction for the dipole mode with a beam charge of 0.1 or $1 \mathrm{nC}$. However, the emittance evolution of the dipole mode with $0 \mathrm{nC}$ beam charge showed higher values in the vertical $(y)$ direction than in the horizontal $(x)$ direction. These phenomena can also arise because the beam is rotated by the solenoid field.

\section{CONCLUSION AND DISCUSSION}

The effect of the multipole mode induced by the asymmetric geometry of an rf gun on emittance evolution was investigated using the Fourier transform technique. In the case of the one-hole model, the emittance in the vertical (y) direction was increased because of the dipole mode. However, the dipole mode can be reduced by adding one more hole in the opposite position. We can also reduce the quadrupole mode by adding two more holes at 90 degrees to the previous two holes. The emittance growth almost disappears only when the dipole and quadrupole modes are suppressed. In conclusion, the effects of the emittance growth due to field asymmetry almost disappeared when four holes were made on the side of the full cell of the rf gun.

In a practical view, if we apply our four-holes model used in the simulation to a real rf gun, the pulsed heating problem can be a considerable issue. But it can be controlled by introducing a rounding shape around the hole entrance at the side of the full cell, proper cooling channels, and strengthened materials such as GlidCop [9,23].

\section{ACKNOWLEDGMENTS}

This work was supported by the National Research Foundation of Korea (NRF) funded by the Ministry of Education, Science and Technology (MEST) (Grant No. 2008-0059842). This work was supported partially by the National Natural Science Foundation of China (Grant No. 10735050).

\section{APPENDIX A}

We used the rf gun model in Ref. [11] as a reference model for simulation. The reference model we used here in simulation has mode separation of $3.41 \mathrm{MHz}$, coupling coefficient of 1.2, and $Q_{0}$ of 9000 [11]. Note the specific dimensions related with the rf gun were determined simply as the same given in Ref. [11]. Any other dimensions can be used for the same study described in this paper. In this case, holes are located at the position of $61 \mathrm{~mm}$ from the cathode which is the center of full cell cavity. They are track shaped with the size of $22.5 \mathrm{~mm} \times 11 \mathrm{~mm}$ as shown in Fig. 12. With any size of the hole, we can study the same effect of the rf asymmetry on the emittance growth. The optimization of the hole size to replace the waveguide coupling hole to the pumping hole will be reported soon [24].

\section{APPENDIX B}

\section{Detail for FFT}

The process of FFT on the field distribution in the rf gun cavity is shown in Fig. 13.

First we get electromagnetic field data on the point corresponding to the $(r, \theta, z)$ coordinate by using 3D high frequency eigenmode solver. Then we separate field distribution into slice in the $z$ direction, that is, transverse field distribution on the $r-\theta$ plane of each $z$ coordinate. Again we separate slice distribution into circular distribution of each $r$ coordinate which is $\theta$ dependent. Field distribution in Fig. 3(a) is the result at the position of $r=9.5 \mathrm{~mm}$, $z=61 \mathrm{~mm}$.

Next, we get the information of magnitude and phase angle of each multipole, such as monopole, dipole, quadrupole, etc. by conducting FFT on $\theta$-dependent

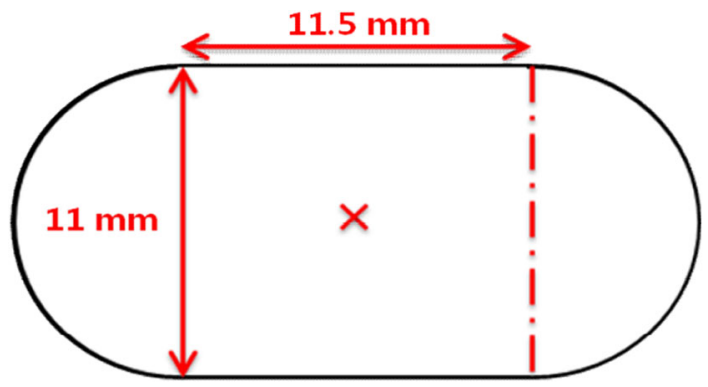

FIG. 12. Shape and size of the hole, which is added to the side of the full cell, used in simulation. 


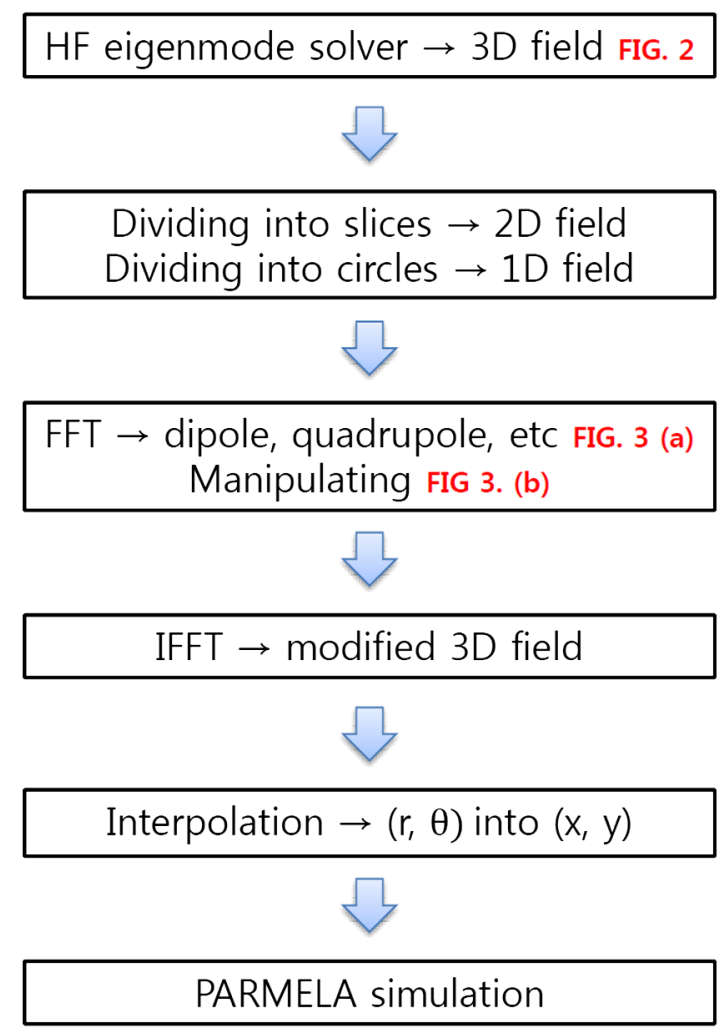

FIG. 13. The process of FFT.

distribution. Then we can manipulate magnitude of each multipole so that we can keep or remove a particular multipole mode. Adding a particular mode with monopole mode and conducting the inverse fast Fourier transform, we get a reconstructed (or manipulated) $\theta$-dependent distribution. Field distribution in Fig. 3(b) is the result of this process. For example, distribution of dipole mode in Fig. 3(b) is the sum of the monopole mode and the dipole mode. By doing this, we can manipulate $\theta$-dependent field distribution in all $z$ coordinates and all $r$ coordinates. Reconstructed field data is the value on the cylindrical coordinate, thus we need to interpolate properly because input field distribution of PARMELA uses the Cartesian coordinate. After sorting field data to fit for PARMELA input order, we get PARMELA input finally.

\section{Detail for simulation procedure}

Input 9 which is Gaussian distribution was used as PARMELA input. The 3D field extracted by the 3D high frequency solver was used as the rf gun cavity field by "cfield" command of PARMELA during the simulation. A solenoid field was inserted by the POISSON command instead of "EM3DField" because we assumed that the solenoid field has cylindrical symmetry. The particle centroid was kicked off the axis by multipole modes. For example, an electron beam was kicked in the vertical ( $y$ ) direction by $14.2 \mathrm{mrad}$. It is also stated at the end of the first paragraph in Sec. IVA.
[1] K. J. Kim, Nucl. Instrum. Methods Phys. Res., Sect. A 275, 201 (1989).

[2] X. Guan, C. Tang, H. Chen, W. Huang, X. He, P. Xu, and R. Li, Nucl. Instrum. Methods Phys. Res., Sect. A 574, 17 (2007).

[3] D. H. Dowell et al., Nucl. Instrum. Methods Phys. Res., Sect. A 528, 316 (2004).

[4] D. T. Palmer, R. H. Miller, H. Winick, X. J. Wang, K. Batchelor, M. Woodle, and I. Ben-Zvi, in Proceedings of the Particle Accelerator Conference, Dallas, TX, 1995 (IEEE, Piscataway, 1995), pp. 982-984.

[5] D. H. Dowell, E. Jongewaard, J. Lewandowski, C. Limborg, Z. Li, J. Schmerge, A. Vlieks, J. Wang, and L. Xiao, SLAC Report No. SLAC-PUB-13401, 2008.

[6] K. Batchelor et al., in Proceedings of the 1990 European Particle Accelerator Conference, Nice, France (Editions Frontiers, Gif-sur-Yvette, France, 1990), p. 541.

[7] J. B. Rosenzweig, S. Anderson, X. Ding, and D. Yu, in Proceedings of the 1999 Particle Accelerator Conference, New York, New York, 1999 (IEEE, Piscataway, NJ, 1999), pp. 2042-2044.

[8] C. Limborg, Z. Li, L. Xiao, J. F. Schmerge, D. Dowell, S. Gierman, E. Bong, and S. Gilevich, Report No. LCLS-TN05-3, 2005.

[9] L. Xiao, R. F. Boyce, D. H. Dowell, Z. Li, C. LimborgDeprey, and J.F. Schmerge, in Proceedings of the 21st Particle Accelerator Conference, Knoxville, 2005 (IEEE, Piscataway, NJ, 2005), pp. 3432-3434.

[10] R. Akre, D. H. Dowell, P. Emma, J. Frisch, S. Gilevich, G. Hays, Ph. Hering, R. Iverson, C. Limborg-Deprey, H. Loos, A. Miahnahri, J. Schmerge, J. Turner, J. Welch, W. White, and J. Wu, Phys. Rev. ST Accel. Beams 11, 030703 (2008).

[11] J. H. Park, S. J. Park, C. Kim, Y. W. Parc, J. H. Hong, J. Y. Huang, D. Xiang, X. Wang, and I. S. Ko, Jpn. J. Appl. Phys. 46, 1751 (2007).

[12] S.I. Moon, J.H. Hong, Y.W. Parc, M.H. Cho, W. Namkung, I. S. Ko, and S. J. Park, Jpn. J. Appl. Phys. 49, 086401 (2010).

[13] J. Hong, S. Moon, Y. W. Parc, M. H. Cho, I. S. Ko, C. Kim, S. J. Park, Y. J. Park, S. H. Kim, and W. Namkung, J. Korean Phys. Soc. 58, 198 (2011).

[14] Y.W. Parc and I. S. Ko, J. Korean Phys. Soc. 54, 1481 (2009).

[15] Z. Li et al., Nucl. Instrum. Methods Phys. Res., Sect. A 558, 168 (2006).

[16] L. Young and J. Billen, Los Alamos National Laboratory Technical Report No. LA-UR-96-1835, 1996.

[17] W. K. H. Panofsky and W. A. Wenzel, Rev. Sci. Instrum. 27, 967 (1956).

[18] M. J. Browman, in Proceedings of the 1993 Particle Accelerator Conference, Washington, DC, 1993 (IEEE, Piscataway, NJ, 1993), pp. 800-802.

[19] J. Hong, M. S. Chae, I. S. Ko, S. I. Moon, and Y. W. Parc, in Proceedings of the 2010 International Particle Accelerator Conference, Kyoto, Japan (ICR, Kyoto, 2010), pp. 1740-1742.

[20] This value is much smaller than the simulated one. The underestimation may be a result of some approximation during the calculation. To check validity of 
our simulation with the 3D input field we used here, we compare the simulation result of 3D input with that of 2D input (POISSON program is used to get the 2D field) which has been already proven by many references $[8,10]$. We carried out 2D simulation of zero charge without a solenoid field so that we can see the pure rf effect. The result of $2 \mathrm{D}$ simulation also is almost the same as the result of $3 \mathrm{D}$ simulation which is not shown in this study. That is, validity of the $3 \mathrm{D}$ input field we used in the simulation is not an issue. Futhermore, the simulation result in this study shows that monopole emittance has linear dependency on bunch length, not square dependency as predicted in Eq. (35) of Ref. [1].

[21] Y. Ding, A. Brachmann, F.-J. Decker, D. Dowell, P. Emma, J. Frisch, S. Gilevich, G. Hays, Ph. Hering, Z. Huang, R. Iverson, H. Loos, A. Miahnahri, H.-D. Nuhn, D. Ratner, J. Turner, J. Welch, W. White, and J. Wu, Phys. Rev. Lett. 102, 254801 (2009).

[22] M. Ferrario et al., Phys. Rev. Lett. 99, 234801 (2007).

[23] D. P. Pritzkau and R. H. Siemann, Phys. Rev. ST Accel. Beams 5, 112002 (2002).

[24] J. H. Hong, I. S. Ko, S. J. Park, and Y. W. Parc (unpublished). 